\title{
Capecitabine metronomic chemotherapy inhibits the proliferation of gastric cancer cells through anti-angiogenesis
}

\author{
FEI YUAN $^{1 *}$, HAILONG SHI $^{2 *}, \mathrm{JUN} \mathrm{JI}^{2}, \mathrm{QU} \mathrm{CAI}^{2}, \mathrm{XUEHUA} \mathrm{CHEN}^{2}$, YINGYAN YU $^{2}$, \\ BINGYA LIU ${ }^{2}$, ZHENGGANG ZHU ${ }^{2,3}$ and $\mathrm{JUN}_{\mathrm{ZHANG}}{ }^{2,3}$ \\ ${ }^{1}$ Department of Pathology, ${ }^{2}$ Department of Surgery, Shanghai Institute of Digestive Surgery and ${ }^{3}$ Department of Clinical \\ Oncology, Ruijin Hospital, Shanghai Jiaotong University School of Medicine, Shanghai 200025, P.R. China
}

Received November 20, 2014; Accepted January 12, 2015

DOI: $10.3892 /$ or.2015.3765

\begin{abstract}
To evaluate the inhibitory effect and mechanism of capecitabine metronomic chemotherapy on gastric cancer cells. In vitro, the effects of 5-fluorouracil ( $\mathrm{Fu})$ metronomic chemotherapy on proliferation, apoptosis, tube formation ability, and angiogenesis were detected. In vivo, Ki-67, CD34 and VEGF were detected by immunohistochemical staining (IHC). Flow cytometry was used to detect the percentage of circulating endothelial progenitors (CEPs), and VEGF and PDGF were detected by ELISA in the peripheral blood of nude mice. The proliferation of the SGC-7901 and AGS gastric cancer cell lines in the metronomic 5-Fu group was decreased compared with the control group in vitro. The total length of the small tubes and tubular junction numbers were significantly lower in the metronomic group than the control group. The VEGF and PDGF levels in the cell culture supernatants were lower in the metronomic group than the control group. Compared with the control group, the CEP percentage was decreased in the peripheral blood of tumor-bearing nude mice following treatment with metronomic 5-Fu or capecitabine chemotherapy. No significant changes were found in the conventional or control group. In the peripheral blood of tumor-bearing nude mice, the VEGF and PDGF levels were decreased in the metronomic groups. Metronomic 5-Fu inhibited the proliferation of gastric cancer cells in vitro and in vivo, and their antitumor effects were non-inferior to those of conventional dose chemotherapy, with mild side effects. Thus, tumor inhibition may be attributed to anti-angiogenesis.
\end{abstract}

Correspondence to: Dr Jun Zhang, Department of Clinical Oncology, Shanghai Institute of Digestive Surgery, Ruijin Hospital, Shanghai Jiaotong University School of Medicine, 197 Ruijin Er Road, Shanghai 200025, P.R. China

E-mail: jun_zj10977@163.com

*Contributed equally

Key words: capecitabine, metronomic chemotherapy, gastric cancer, anti-angiogenesis

\section{Introduction}

Gastric cancer is one of the most common malignant tumors worldwide, and its incidence is fourth among malignant tumors, ranking second in the leading causes of death (1). Surgical resection was the standard treatment for early gastric cancer. In China, the majority of patients are diagnosed at an advanced stage, representing lost opportunities for radical surgery. Systemic chemotherapy is the main treatment for such patients (2). Traditional chemotherapy for gastric cancer includes large doses of cytotoxic drugs that last 2-3 weeks. However, although these agents have a tumor suppressive effect, they are accompanied by heavy toxic side effects. In addition, their high-dose and intermittent-dosing patterns make remnants of tumor cells grow between chemotherapeutic intervals, inducing acquired drug resistance and resulting in treatment failure (3). To avoid problems associated with traditional chemotherapy, attempts have been made to switch from 'maximum tolerable dose (MTD)' to 'minimal effective dose (MED)' as a new delivery mode to reduce toxicity and for synergistic purposes.

Bertolini et al (3) demonstrated 'metronomic chemotherapy' as a mode of drug administration with sustainability, using low-dose high-frequency cytotoxic drugs (4). In breast, ovarian, prostate, and colorectal cancer animal models and clinical trials, metronomic chemotherapy was demonstrated to have superior efficacy to MTD, and the side effects were significantly less than those of traditional chemotherapy (5-7). Preclinical studies confirmed that metronomic chemotherapy selectively affected tumor-derived vascular endothelia and angiogenesis $(8,9)$. However, whether metronomic chemotherapy exert an anti-angiogenesis effect has not yet been reported. Notably, the VEGF-neutralizing antibody bevacizumab was unsuccessful as a first-line treatment for gastric cancer in two large clinical studies, while the anti-VEGFR monoclonal antibody ramucizumab demonstrated positive results during and after second-line treatment for gastric cancer, indicating that anti-angiogenesis was the key factor affecting treatment success or failure $(10,11)$.

Fluorouracil (Fu)-based chemotherapy has been widely used as chemotherapy for gastric cancer (12). Large clinical studies of REAL-2 and ML17032 demonstrated the value of oral $\mathrm{Fu}$ drugs, including capecitabine, as a first-line treatment 
of gastric cancer $(13,14)$. The CLASSIC and ARTIST studies demonstrated the value of postoperative adjuvant chemotherapy (radiotherapy) for gastric carcinoma $(15,16)$. In addition, oral administration more easily accommodated smaller doses, as compared to high-frequency 'metronomic chemotherapy'. A preliminary study from our research group found that metronomic capecitabine inhibited cell proliferation in colon cancer cells, and its tumor suppressor effect was achieved by anti-angiogenesis (9).

The aim of the present study was to evaluate whether capecitabine chemotherapy with a metronomic pattern may play an inhibitory role in the growth of gastric cancer cells in vitro and in vivo and whether anti-angiogenesis was involved in this inhibitory effect.

\section{Materials and methods}

Ethics statement. The human gastric cancer cell lines and human umbilical vein endothelial cells (HUVEC) were conserved by the Shanghai Institute of Digestive Surgery as described previously $(9,17)$. The use of these cell lines was approved by the Ethics Committee of Ruijin Hospital, Shanghai Jiaotong University School of Medicine. The mouse experiments were approved by the Animal Care and Use Committee and conducted in accordance with the Guide for the Care and Use Laboratory Animals of Ruijin Hospital, Shanghai Jiaotong University School of Medicine (Shanghai, China).

Cell culture. GES-1, SNU-1, SNU-16, MKN-45, KATOIII, BGC-823, AGS, SGC-7901, NCI-N87, MGC-803, MKN-28 and HUVEC human gastric cancer cell lines were cultured in RPMI-1640 medium (Gibco-BRL, Carlsbad, CA, USA) supplemented with $10 \%$ fetal bovine serum (FBS; Gibco-BRL) at $37^{\circ} \mathrm{C}$ with $5 \% \mathrm{CO}_{2}$ and saturated humidity.

Western blot analysis. Total protein was extracted by using the Mammalian Protein Extraction reagent (Pierce, Rockford, IL, USA) mixed with protease inhibitor cocktail (Sigma, St. Louis, MO, USA). Samples with an equal amount of total protein $(100 \mu \mathrm{g})$ were fractionated by $10 \%$ SDS-PAGE and transferred to PVDF (Bio-Rad, Hercules, CA, USA), membranes. The PVDF membranes were blocked by TBST buffer with $5 \%$ skim milk for $2 \mathrm{~h}$, and incubated at $4^{\circ} \mathrm{C}$ with primary antibodies overnight, TP and TS antibodies (1:1,000; both from Abcam, Cambridge, MA, USA). Fluorescent secondary antibodies (1:15,000; Li-COR Biosciences, Lincoln, NE, USA) and Infrared Imaging System (Li-COR Biosciences) were used to visualize the protein bands of interest.

CCK- 8 cell proliferation assay. The logarithmic growth of SGC-7901 and AGS cells was seeded at $1 \times 10^{3}$ cells/well in a 96-well plate and incubated overnight with different concentrations of 5-Fu (Shanghai Xudong Haipu Pharmaceutical Co., Ltd., Shanghai, China) (5, 10, 20, 40, 80, 160, 320, and $640 \mu \mathrm{g} / \mathrm{l})$ which were added and cultured for 5 days. The drug-containing culture medium was replaced every $24 \mathrm{~h}$. The $\mathrm{OD}_{450}$ values were detected every $24 \mathrm{~h}$ using Cell Counting kit-8 (Dojindo, Kumamoto, Japan) and the inhibitory rate was calculated. $\mathrm{IC}_{25}$ and $\mathrm{IC}_{50}$ values of 5-Fu for the SGC-7901 and
AGS cells were calculated according to a 48-h inhibitory rate for the conventional in vitro and metronomic doses, respectively. Based on the results, different doses of 5-Fu (standard dose, $20 \mu \mathrm{g} / 1$ once; metronomic chemotherapy dose, $5 \mu \mathrm{g} / 1$ per day) were used to treat the gastric cancer cells for 5 days, and the effects of 5-Fu metronomic chemotherapy on the proliferation of gastric cancer cells in vitro were observed. Each concentration was repeated three times.

Analysis of apoptosis by flow cytometry. The cell density was adjusted to $1 \times 10^{5}$ cells/well in a 24-well plate, and standard and metronomic doses of 5 -Fu $(20$ or $5 \mu \mathrm{g} / \mathrm{l})$ were administered. The cells were collected after 24 hand-stained with FITC-conjugated Annexin V (BD Biosciences, Bedford, MA, USA). Propidium iodide (BD Biosciences) was subsequently added, and a flow cytometric analysis was performed using FACS (Becton-Dickinson, Bedford, MA, USA).

HUVEC tube formation assay. Matrigel (100 $\mu \mathrm{l})$ was added to each well in a 96-well plate, until the glue completely solidified, and 5x10 HUVECs were then added to each well. Standard and metronomic doses of $5-\mathrm{Fu}(20$ or $5 \mu \mathrm{g} / \mathrm{l})$ were used to treat the SGC-7901 and AGS cell culture supernatants (200 $\mu \mathrm{l}$ were added, respectively). The cells were incubated at $37^{\circ} \mathrm{C}$ with $5 \% \mathrm{CO}_{2}$ for $8 \mathrm{~h}$, and non-adherent cells were eluted with PBS. Under high magnification, three randomly-selected images were assessed for the following: the number of tubular forms, total length, and the number of connection points. Each concentration was repeated three times.

Angiogenic factors detection by enzyme-linkedimmunosorbent assay. The SGC-7901 and AGS cells were seeded in a 24-well plate $\left(1 \times 10^{4}\right.$ cells/well). After cell adherence, the suspension was centrifuged, and the supernatant was discarded. Standard and metronomic doses of 5 - $\mathrm{Fu}(20$ or $5 \mu \mathrm{g} / \mathrm{l})$ were added, and the cells were cultured at $37^{\circ} \mathrm{C}$ with $5 \% \mathrm{CO}_{2}$ for $8 \mathrm{~h}$. The cell culture supernatant was then collected for VEGF (MAB293; R\&D Systems Inc., Minneapolis, MN, USA) and PDGF (900K04; PeproTech, Rocky Hill, NJ, USA) detection using an ELISA kit.

Establishment of gastric cancer xenografts and tissue collection. Male Balb/c nude mice, 4-6 weeks of age, with a body weight of $15-20 \mathrm{~g}$, were provided by the Research Center of Experimental Medicine, Shanghai Jiaotong University School of Medicine Affiliated Ruijin Hospital. Prior to performing the experiment, the animals were placed in separate cages for 1 week to adapt to the new environment. The SGC-7901 cell suspension was adjusted to a cell density of $1 \times 10^{7} / \mathrm{ml}$, and the nude mice were subcutaneously inoculated with a $100-\mu 1$ suspension. Administration of the therapy was initiated when the subcutaneous nodules were $\sim 2 \mathrm{~mm}$ in diameter. The nude mice were randomly divided into the following groups: i) control group, intraperitoneally injected with normal saline (NS); ii) 5-Fu conventional dose group (5-Fu MTD group), intraperitoneally injected with $50 \mathrm{mg} / \mathrm{kg}$, twice per week for 2 weeks, with an 1-week discontinuation for 6 weeks (18); iii) 5-Fu metronomic group (5-Fu LDM group), intraperitoneally injected with $15 \mathrm{mg} / \mathrm{kg}$, twice a week for 6 weeks; iv) capecitabine (Roche Company, Shanghai, China) 
A

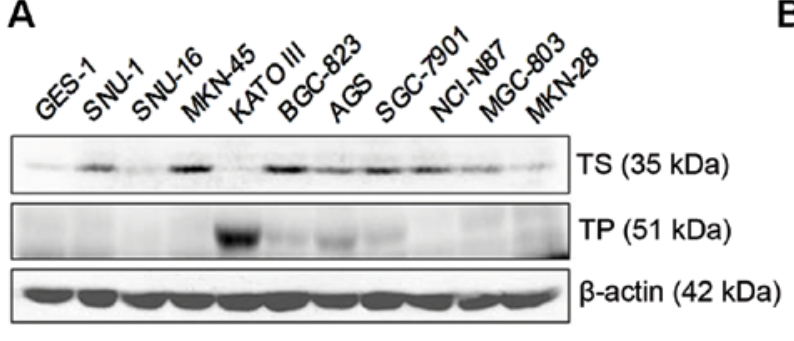

$B$

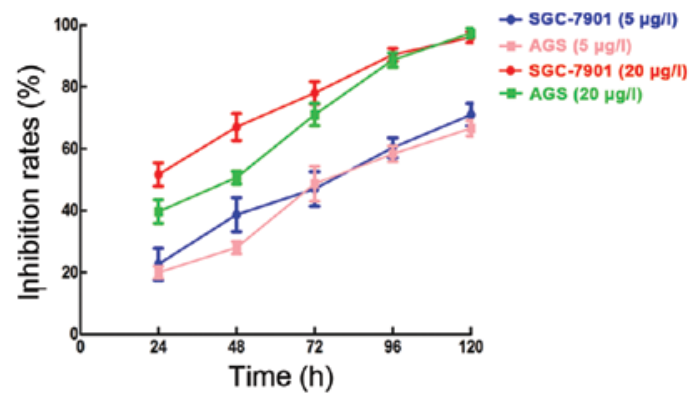

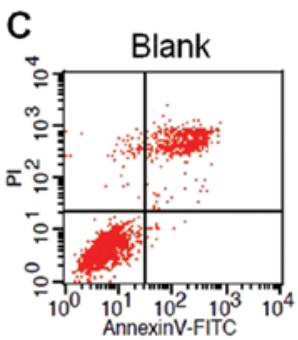
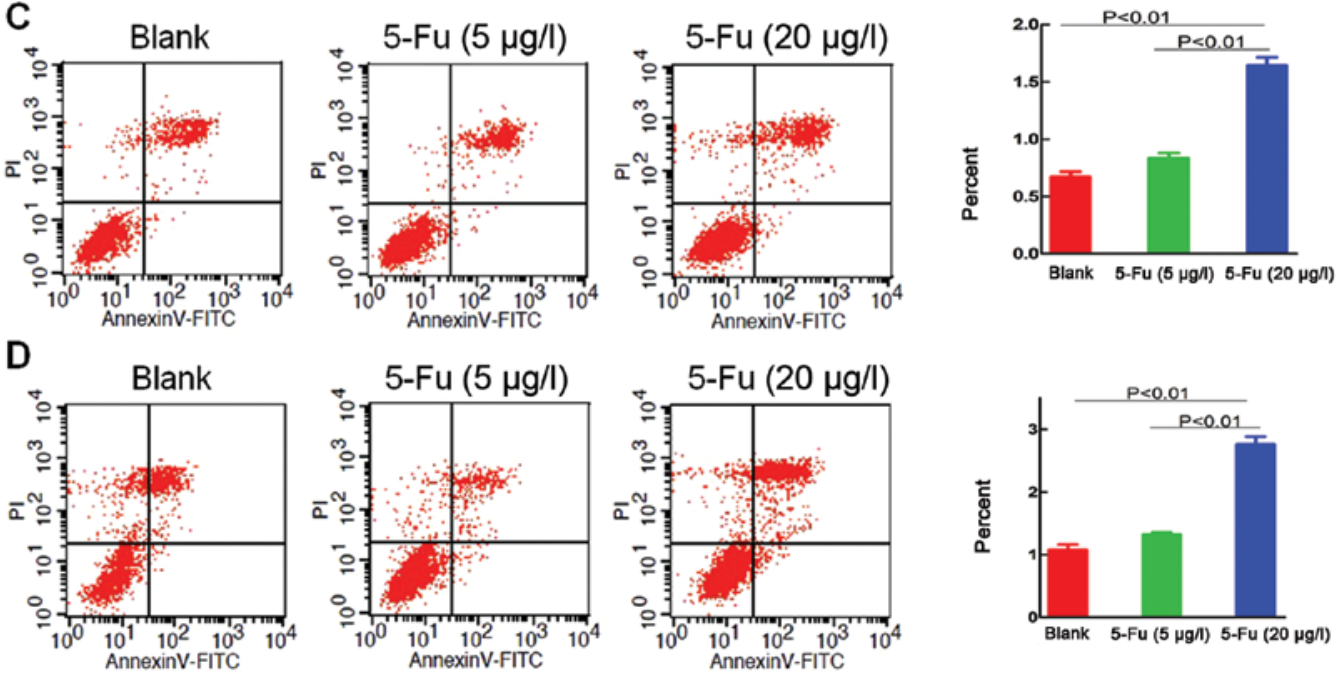

Figure 1. (A) Western blot analysis screening of gastric cancer cell lines. (B) In vitro proliferation of gastric cancer cells. Detection of apoptosis in gastric cancer cells (C: SGC-7901; D: AGS).

conventional dose (capecitabine MTD group), IG $500 \mathrm{mg} / \mathrm{kg}$, twice per week for 2 weeks, with a 1-week discontinuation for 6 weeks (19); and v) capecitabine metronomic group (capecitabine LDM group), intragastric administration at $200 \mathrm{mg} / \mathrm{kg}$, twice a week for 6 weeks. From the beginning of drug administration, a Vernier caliper was used to measure the tumor length/diameter (L) and short track (W) every 7 days to calculate the tumor volume $(\mathrm{V})$ according to the following formula: $\mathrm{V}=(\mathrm{W}+\mathrm{L}) /(2 \times \mathrm{W} \times \mathrm{L} \times 0.5236)$. A tumor growth curve was drawn. The nude mice were executed after 6 weeks of treatment, and the tumor tissue and peripheral blood samples were collected.

Immunohistochemical staining (IHC). The nude mouse transplantation tumor was fixed with $10 \%$ formaldehyde. After HE staining for tumor confirmation, the immunohistochemical staining was performed on $4-\mu \mathrm{m}$ sections following the EnVision two-step procedure of Dako REAL ${ }^{\mathrm{TM}}$ Envision $^{\mathrm{TM}}$ Detection system (Dako, Denmark). The slides were incubated with the primary antibodies for Ki-67 (1:50; Dako), CD34 (1:150; Abcam) and VEGF (1:200; Santa Cruz Biotechnology, Inc., Santa Cruz, CA, USA), respectively. The HRP-labeled antibody to rabbit and mouse immunoglobulin was used as secondary antibodies. The slides were visualized by diaminobenzidine under a microscope. For selection of three high-power fields to count for microvascular density (MVD), the staining result criteria were as follows: a tumor with yellowish-brown granules in the cell nucleus was positive for Ki-67 staining; a tumor with brownish-yellow granules was positive for VEGF staining; and a tumor with brown particles in the cell plasma was positive for CD34 staining (20).

Measurement of circulating endothelial progenitors (CEPs) by flow cytometry. The peripheral blood cells in the nude mice were treated with an EDTA anticoagulant (BD Biosciences). Following erythrocyte lysis, the dead cells, platelets, and cell debris were washed away. FCS500 MC (BD Biosciences) was used to detect cell suspension. The monoclonal antibodies CD117, Flk-1 (VEGFR-2), and Sca-1 (eBioscience, San Diego, CA, USA) were used to identify CEP subsets. From each specimen, we obtained at least $5 \times 10^{4}$ cells. With the CEP counts, we collected enough cells in the window ( $>50$, typically 100-200) to determine the percentage of stained cells.

Statistical analysis. The SPSS software (v13.0; SPSS Inc., Chicago, IL, USA) was used for statistical analysis. Data are presented as mean \pm standard deviation, and differences between the groups were compared using ANOVA and the Mann-Whitney U test. $\mathrm{P}<0.05$ was considered significant.

\section{Results}

Effects of metronomic chemotherapy on the proliferation of gastric cancer cells in vitro. The antitumor effects of capecitabine partly depend on TP enzymes, which inside the cells convert 5-Fu precursor to 5-Fu and plays a role for the target enzyme of TS. Therefore, the AGS and SGC-7901 gastric cancer cell lines, which have a high expression of TP 

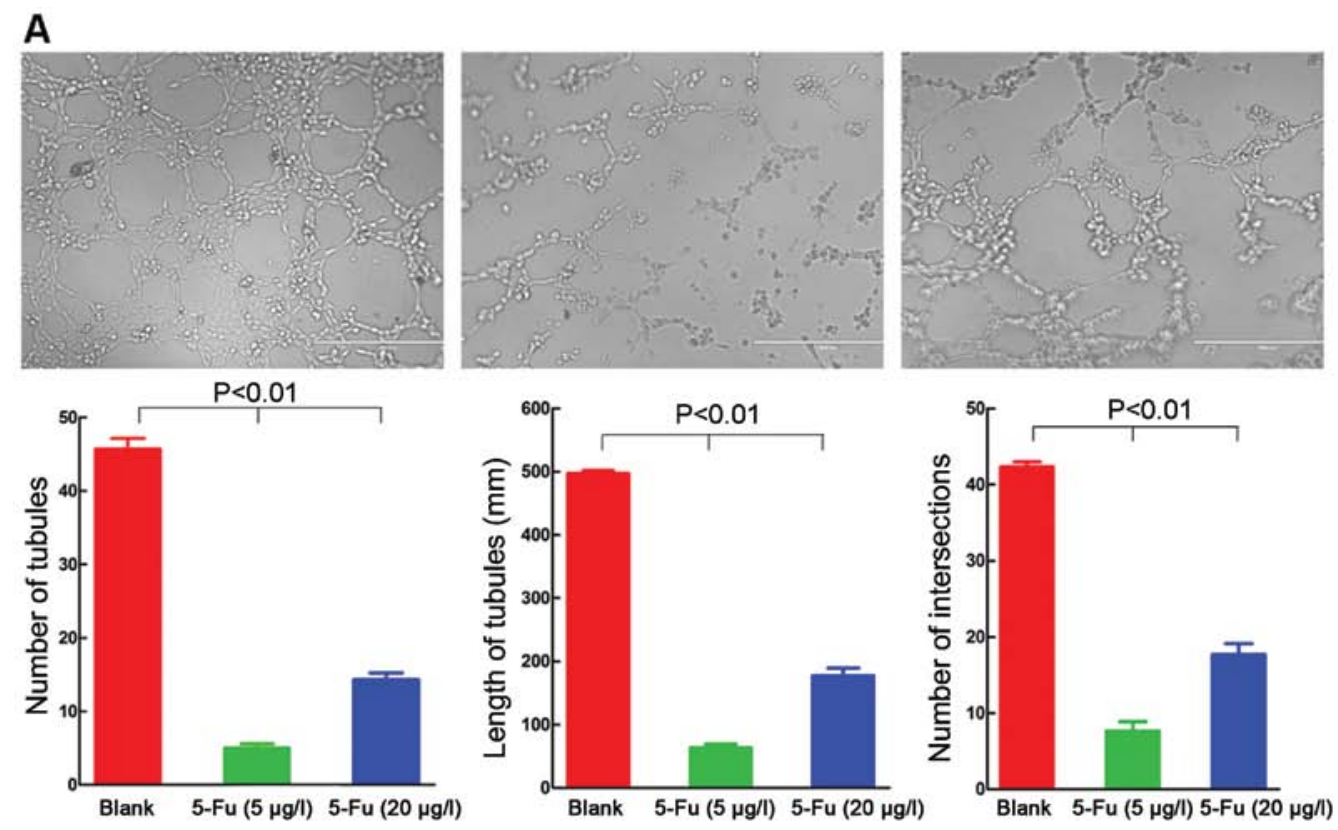

B
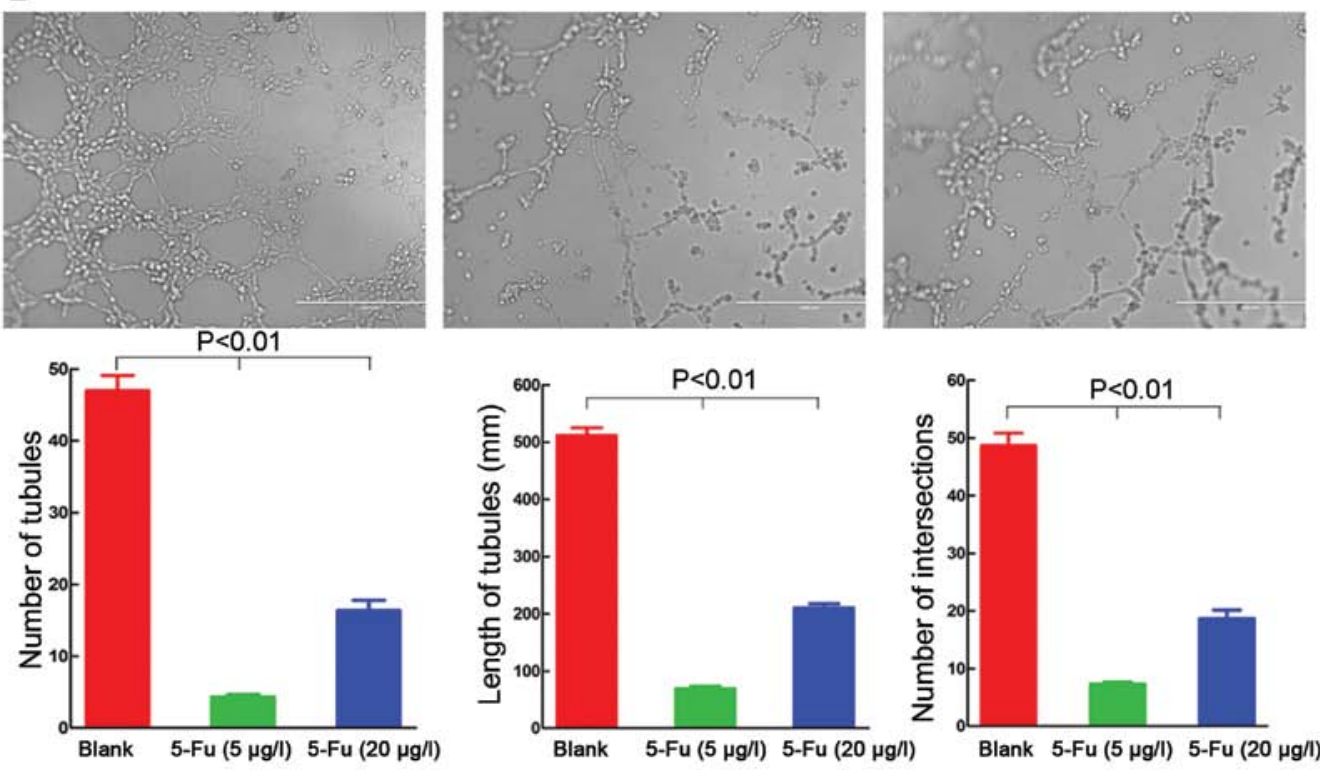

Figure 2. HUVEC tube formation assay in vitro (A: SGC-7901; B: AGS).

and a low expression of TS were screened by western blot analysis for subsequent experiments (Fig. 1A). The proliferation inhibitory rates of 5-Fu at $48 \mathrm{~h}$, as well as the $\mathrm{IC}_{25}$ and $\mathrm{IC}_{50}$ of 5-Fu, for SGC-7901 and AGS were calculated (Table I). The in vitro concentrations of the conventional and metronomic doses were confirmed (20 and $5 \mu \mathrm{g} / 1$, respectively).

The cell proliferation results showed that the $5-\mathrm{Fu}$ metronomic dose significantly inhibited proliferation of the SGC-7901 and AGS gastric cancer cell lines in vitro, as compared with the control group, and after 5 days, the inhibitory rates in the SGC-7901 and AGS groups were 71 \pm 4.0 and $66.6 \pm 2 \%$, respectively. At 5 days, the $5-\mathrm{Fu}$ conventional dose had inhibitory rates that were close to $100 \%$ in the SGC-7901 and AGS groups, and almost all the cells were dead (Fig. 1B).

Effect of metronomic chemotherapy on the apoptosis of gastric cancer cells in vitro. Compared with the SGC-7901 control
Table $\mathrm{I} . \mathrm{IC}_{25}$ and $\mathrm{IC}_{50}$ of 5-Fu for gastric cancer cells.

\begin{tabular}{lll}
\hline Gastric cancer cells & $\mathrm{IC}_{25}(\mu \mathrm{g} / \mathrm{l})$ & $\mathrm{IC}_{50}(\mu \mathrm{g} / \mathrm{l})$ \\
\hline SGC-7901 & $6.71 \pm 0.83$ & $18.59 \pm 1.27$ \\
AGS & $5.37 \pm 0.73$ & $16.45 \pm 1.22$ \\
\hline
\end{tabular}

Fu, fluorouracil.

group $(0.67 \pm 0.08 \%)$, the apoptotic index for the 5-Fu metronomic group was $0.83 \pm 0.08 \%(\mathrm{P}=0.087)$, and the apoptotic index for the $5-\mathrm{Fu}$ conventional dose group was $1.64 \pm 0.13 \%$ $(\mathrm{P}<0.01$, Fig. 1C).

In the control AGS cells, the apoptotic index was $1.08 \pm 0.15 \%$, and in the 5 -Fu metronomic group, the apoptotic 

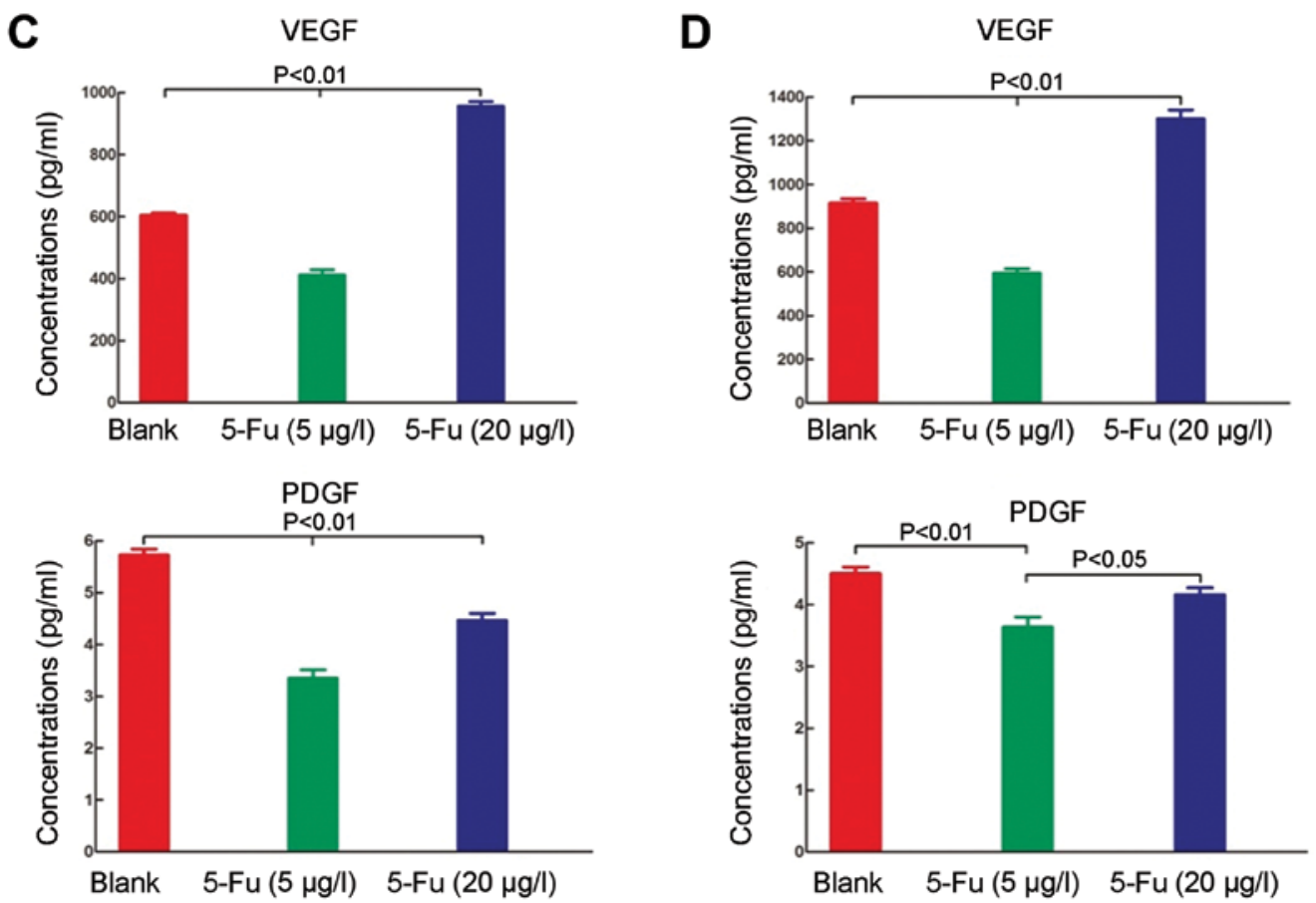

Figure 2 Continued. Angiogenesis-related factor detection in gastric cancer cell culture supernatant using ELISA (C: SGC-7901; D: AGS). HUVEC, human umbilical vein endothelial cells.

index was $1.32 \pm 0.07 \%(\mathrm{P}=0.105)$. The apoptotic index was $2.76 \pm 0.21 \%$ for the 5 -Fu conventional dose group $(\mathrm{P}<0.01$; Fig. 1D). These results indicated that a conventional dose of 5-Fu promoted the apoptosis of gastric cancer cells in vitro. However, metronomic chemotherapy had no effect on the apoptosis of gastric cancer cells in vitro. Different doses of 5-FU induced the apoptosis of gastric cancer cells in different ways. The standard doses showed a direct cytotoxic effect on gastric cancer cells, while metronomic chemotherapy inhibited the proliferation of gastric cancer cells in vitro without any effect on cell apoptosis.

Effect of metronomic chemotherapy on the HUVEC tube formation ability in vitro. Image-Pro Plus Professional software was used to measure the number of small tubes, total length, and number of connection points between HUVEC tubes. Compared with those in the control group, the number and total length of the small tubes, as well as the number of tubular junctions, in the 5-Fu metronomic and conventional dose groups were significantly reduced $(\mathrm{P}<0.01)$. Compared with the 5-Fu conventional dose group, the number and total length of the small tubes, as well as the number of tubular junction numbers in the 5-Fu metronomic group were significantly reduced $(\mathrm{P}<0.01$; Fig. $2 \mathrm{~A}$ and $\mathrm{B}$, Table II). This indicated that 5-Fu significantly inhibited HUVEC tube formation ability in vitro, and the inhibitory effect of 5-Fu metronomic was evident.

Effect of metronomic chemotherapy on the secretory angiogenesis-related factors of gastric cancer cells. The ELISA results showed that the VEGF concentration in the SGC-7901 cell culture supernatants in the control group was $605.07 \pm 10.75 \mathrm{pg} / \mathrm{ml}$, while the VEGF concentrations were $411.53 \pm 28.79 \mathrm{pg} / \mathrm{ml}(\mathrm{P}<0.01)$ and $956.43 \pm 25.21 \mathrm{pg} / \mathrm{ml}(\mathrm{P}<0.01)$ in the 5-Fu metronomic and conventional dose groups, respectively. The VEGF concentration in the AGS cell culture supernatants in the control group was $914.93 \pm 35.02 \mathrm{pg} / \mathrm{ml}$, while the VEGF concentrations were $594.60 \pm 34.99 \mathrm{pg} / \mathrm{ml}$ $(\mathrm{P}<0.01)$ and $1300.23 \pm 70.29 \mathrm{pg} / \mathrm{ml}(\mathrm{P}<0.01$; Fig. $2 \mathrm{C})$ in the 5 -Fu metronomic and conventional dose groups, respectively.

The PDGF concentration in the SGC-7901 cell culture supernatants in the control group was $5.73 \pm 0.20 \mathrm{ng} / \mathrm{ml}$, while the VEGF concentrations were $3.35 \pm 0.28 \mathrm{ng} / \mathrm{ml}(\mathrm{P}<0.01)$ and $4.47 \pm 0.23 \mathrm{ng} / \mathrm{ml}(\mathrm{P}<0.01)$ in the $5-\mathrm{Fu}$ metronomic and conventional dose groups, respectively (Fig. 2C). The PDGF concentration in the AGS cell culture supernatants in the control group was $4.50 \pm 0.18 \mathrm{ng} / \mathrm{ml}$, while the PDGF concentrations were $3.64 \pm 0.28 \mathrm{ng} / \mathrm{ml}(\mathrm{P}<0.01)$ and $4.16 \pm 0.20 \mathrm{ng} /$ $\mathrm{ml}(\mathrm{P}=0.11)(\mathrm{P}<0.05$, Fig. $2 \mathrm{D})$ in the $5-\mathrm{Fu}$ metronomic and conventional dose groups, respectively.

These results indicated that 5-Fu metronomic chemotherapy significantly inhibited the gastric secretions of VEGF and PDGF in vitro and that conventional doses of 5-Fu had an anti-VEGF-secretion effect on gastric cancer cells.

Effect of metronomic chemotherapy on gastric cancer xenografts. Compared with the control group, the tumor growth rates in gastric cancer xenografts were significantly decreased. The 5-Fu and capecitabine metronomic groups demonstrated no significant differences, as compared with the conventional dose groups. After drug treatment for 6 weeks, the tumor volume in the control group was $2172.81 \pm 264.44 \mathrm{~mm}^{3}$, which was significant compared with the 5 -Fu conventional dose group $\left(1239.16 \pm 154.25 \mathrm{~mm}^{3} ; \mathrm{P}<0.01\right)$ and $5-\mathrm{Fu}$ metronomic group $\left(1369.74 \pm 459.73 \mathrm{~mm}^{3} ; \mathrm{P}<0.01\right)$. However, no significant difference was found between the 5 -Fu conventional dose and metronomic groups $(\mathrm{P}=0.531)$. The capecitabine conventional dose and metronomic groups demonstrated a 
Table II. HUVEC tubule formation ability in vitro.

\begin{tabular}{lccccccc}
\hline & \multicolumn{3}{c}{ SGC-7901 } & & \multicolumn{3}{c}{ AGS } \\
\cline { 2 - 3 } \cline { 8 - 9 } Variables & Blank & 5 -Fu $(5 \mu \mathrm{g} / \mathrm{l})$ & 5-Fu $(20 \mu \mathrm{g} / \mathrm{l})$ & & Blank & 5-Fu (5 $\mu \mathrm{g} / \mathrm{l})$ & 5-Fu $(20 \mu \mathrm{g} / \mathrm{l})$ \\
\hline No. of tubules & $45.7 \pm 2.5$ & $5.0 \pm 1.0$ & $14.3 \pm 1.5$ & & $47.0 \pm 3.6$ & $4.3 \pm 0.6$ & $16.3 \pm 2.5$ \\
Tubule length (mm) & $496.9 \pm 8.1$ & $63.4 \pm 10.1$ & $177.2 \pm 20.7$ & & $511.8 \pm 23.3$ & $69.5 \pm 6.8$ & $210.5 \pm 12.4$ \\
No. of intersections & $42.3 \pm 1.2$ & $7.7 \pm 2.1$ & $17.7 \pm 2.5$ & & $48.7 \pm 3.8$ & $7.3 \pm 0.6$ & $18.7 \pm 2.5$ \\
\hline
\end{tabular}

Fu, fluorouracil; HUVEC, human umbilical vein endothelial cells.

significant a significant difference of $1339.71 \pm 430.40$ and $1560.24 \pm 186.44 \mathrm{~mm}^{3}$, respectively, as compared with the control group $(\mathrm{P}<0.01)$. However, no significant difference was found between the capecitabine conventional dose and metronomic groups $(\mathrm{P}=0.295$; Fig. 3A).

After a 6-week drug treatment, the tumor weight in the control group was $3.02 \pm 0.58 \mathrm{~g}$, which was statistically different from that of the 5-Fu conventional dose $(1.24 \pm 0.31 \mathrm{~g} ; \mathrm{P}<0.01)$ and metronomic groups $(1.50 \pm 0.56 \mathrm{~g} ; \mathrm{P}<0.01)$. However, no significant difference was found between the 5 -Fu conventional dose and metronomic groups $(\mathrm{P}=0.415)$. The capecitabine conventional dose and metronomic groups were $1.60 \pm 0.16 \mathrm{~g}$ $(\mathrm{P}<0.01)$ and $1.88 \pm 0.60 \mathrm{~g}(\mathrm{P}<0.01)$, respectively. However, no significant difference was found between the capecitabine conventional dose and metronomic groups ( $\mathrm{P}=0.384$; Fig. 3B).

The tumor weight in the control group was $28.82 \pm 2.70 \mathrm{~g}$, which was statistically different from that of the $5-\mathrm{Fu}$ conventional dose $(20.32 \pm 3.49 \mathrm{~g} ; \mathrm{P}<0.01)$ and metronomic groups $(23.54 \pm 2.22 \mathrm{~g} ; \mathrm{P}<0.01)$. The tumor weight in the 5 -Fu conventional dose group was significantly decreased, as compared with the 5-Fu metronomic group $(\mathrm{P}<0.05)$. The capecitabine conventional dose and metronomic groups were $24.38 \pm 0.81 \mathrm{~g}(\mathrm{P}<0.01)$ and $27.60 \pm 1.09 \mathrm{~g}$, respectively $(\mathrm{P}=0.41)$. The capecitabine conventional dose group was statistically different, as compared with the capecitabine metronomic group $(\mathrm{P}<0.05$; Fig. 3C), indicating that metronomic chemotherapy had a lower toxicity than conventional dose chemotherapy in the tumor-bearing nude mice.

Effect of metronomic chemotherapy on the proliferation and apoptosis of gastric cancer xenografts. IHC revealed that the $\mathrm{Ki}-67$ expression was significantly lower in the 5-Fu conventional dose and metronomic groups than the control group (57.67 $\pm 2.52,29.67 \pm 2.08$ vs. $85 \pm 4.00 \%$, respectively; $\mathrm{P}<0.01$ ). The 5-Fu metronomic group was significantly lower than the 5 -Fu conventional dose group $(\mathrm{P}<0.01)$. The Ki-67 expression in the capecitabine conventional dose and metronomic groups was also significantly lower than that of the control group $(39.67 \pm 2.52,30.67 \pm 4.73$ vs. $85 \pm 4.00 \%$, respectively; $\mathrm{P}<0.01)$. The capecitabine metronomic group was significantly lower than the capecitabine conventional dose group $(\mathrm{P}<0.01$; Fig. 3D).

TUNEL staining showed that the 5-Fu and capecitabine conventional dose groups were significantly increased. However, tumor apoptosis in the 5-Fu and capecitabine metronomic groups demonstrated no significant increase (Fig. 3D), indicating that conventional doses of 5-Fu and capecitabine promoted apoptosis in gastric cancer cells but that $\mathrm{Fu}$ and capecitabine metronomic chemotherapy had no obvious apoptotic effect.

Effect of metronomic chemotherapy on the expression of $M V D$ and VEGF in gastric cancer xenografts in nude mice. The IHC results showed that the MVD in the control group was $5.7 \pm 1.2$, while the MVDs in 5-Fu conventional dose and metronomic groups were $4.7 \pm 1.5(\mathrm{P}=0.365)$ and $3.0 \pm 1.0$ $(\mathrm{P}<0.05)$, respectively. The MVDs in the capecitabine conventional dose and metronomic groups were $4.7 \pm 1.2(\mathrm{P}=0.365)$ and $2.7 \pm 1.5(\mathrm{P}<0.05$, Fig. $3 \mathrm{D})$, respectively, indicating that 5-Fu and capecitabine metronomic doses decreased the MVD in the gastric cancer xenografts, but conventional 5-Fu and capecitabine metronomic chemotherapy had no obvious effect on the MVD in gastric cancer xenografts.

The VEGF expression in the 5-Fu and capecitabine conventional dose groups was higher than that of the control group. However, VEGF expression in the 5-Fu and capecitabine metronomic groups was lower than that of the control group, indicating that $5-\mathrm{Fu}$ and capecitabine metronomic chemotherapy reduced VEGF expression in the gastric cancer xenografts. By contrast, 5-Fu and capecitabine conventional doses increased the VEGF expression (Fig. 3D).

Effect of metronomic chemotherapy on the CEPs in gastric cancer xenografts. The flow cytometry results showed that the CEP percentage in the tumor-bearing nude mice in the control group was $32.12 \pm 4.11 \%$ and that the CEP percentages in the 5-Fu conventional dose and metronomic groups were $29.18 \pm 1.61 \%(\mathrm{P}=0.526)$ and $18.94 \pm 1.45 \%(\mathrm{P}<0.05)$, respectively. The capecitabine conventional dose and metronomic groups were $27.08 \pm 4.89 \%(\mathrm{P}=0.286)$ and $20.15 \pm 2.47 \%$ $(\mathrm{P}<0.05$; Fig. 4A), respectively, suggesting that the 5-Fu and capecitabine metronomic groups had reduced CEP percentages in the peripheral blood endothelial progenitor cells and that the 5-Fu and capecitabine conventional doses had no significant effect.

Effect of metronomic chemotherapy on angiogenesis-related factors in the peripheral blood of gastric cancer-bearing nude mice. The ELISA test showed that the VEGF level in the peripheral blood of nude mice in the control group was $75.63 \pm 1.24 \mathrm{pg} / \mathrm{ml}$. The VEGF levels in the 5 -Fu conventional dose and metronomic groups were $87.00 \pm 0.92 \mathrm{pg} / \mathrm{ml}$ 

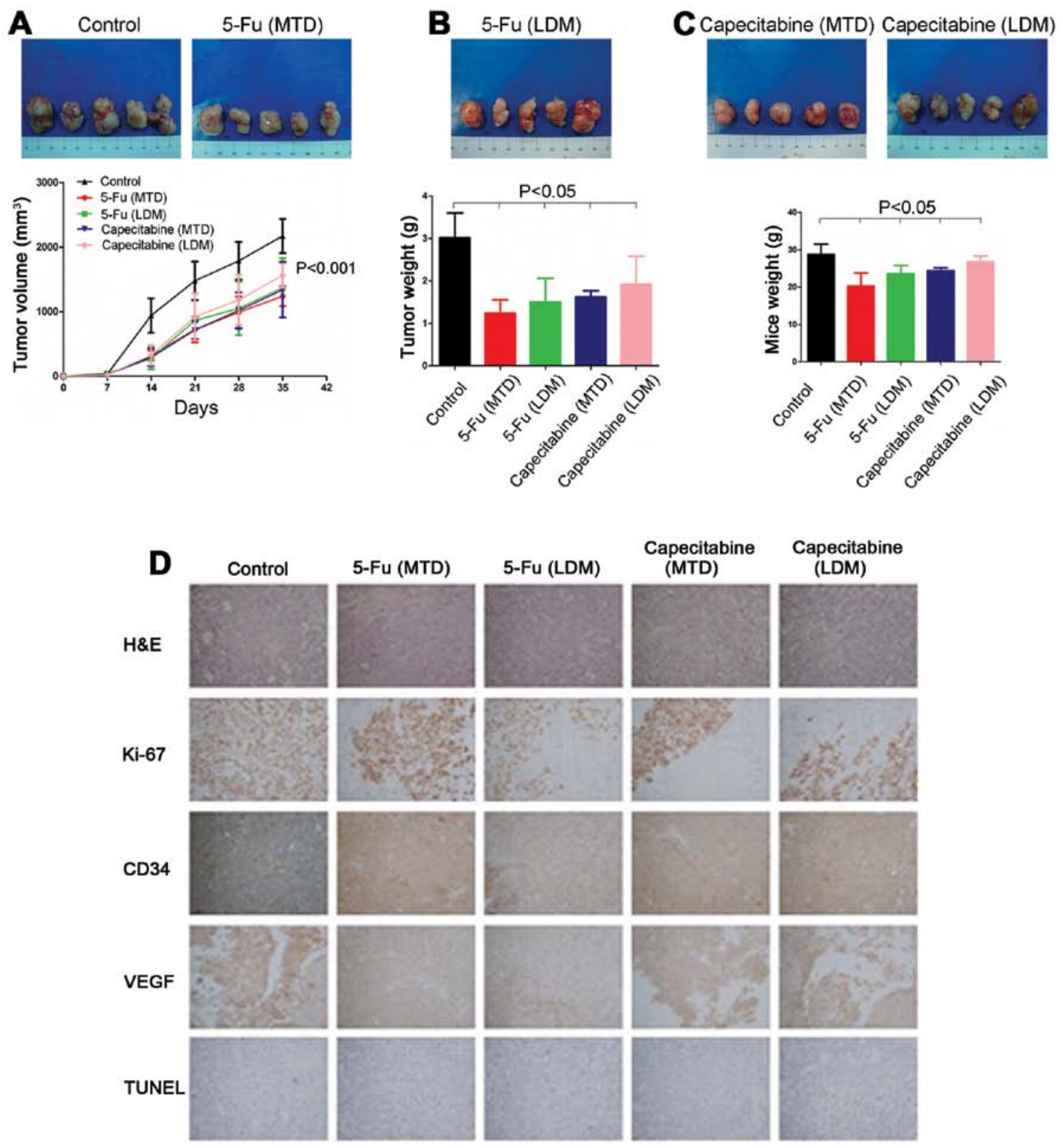

Figure 3. (A) Tumor growth curve. (B) Tumor weight of the SGC-7901 gastric cancer subcutaneous xenografts. (C) Body weight changes for SGC-7901 gastric cancer-bearing nude mice after a 6-week drug treatment. (D) Immunohistochemical staining of SGC-7901 gastric cancer cells in the transplanted tumors of nude mice (x200). IHC, immunohistochemical staining.

$(\mathrm{P}<0.01)$ and $68.32 \pm 4.58 \mathrm{pg} / \mathrm{ml}(\mathrm{P}<0.05)$, respectively. The capecitabine conventional dose and metronomic groups were $94.61 \pm 4.21 \mathrm{pg} / \mathrm{ml}(\mathrm{P}<0.01)$ and $65.39 \pm 4.44 \mathrm{pg} / \mathrm{ml}(\mathrm{P}<0.01$; Fig. 4B), respectively.

The PDGF level in the peripheral blood of the nude mice in the control group was $38.20 \pm 1.43 \mathrm{pg} / \mathrm{ml}$. The PDGF levels in the 5-Fu conventional dose and metronomic groups were $38.34 \pm 1.28 \mathrm{pg} / \mathrm{ml}(\mathrm{P}=0.931)$ and $31.03 \pm 1.46 \mathrm{pg} / \mathrm{ml}$ $(\mathrm{P}<0.01)$, respectively. The capecitabine conventional dose and metronomic groups were $47.00 \pm 3.34 \mathrm{pg} / \mathrm{ml}(\mathrm{P}<0.01)$ and $28.46 \pm 1.28 \mathrm{pg} / \mathrm{ml}(\mathrm{P}<0.01$; Fig. $4 \mathrm{C})$, respectively.

These results suggested that $5-\mathrm{Fu}$ and capecitabine metronomic chemotherapy reduced the peripheral blood levels of VEGF and PDGF in the gastric cancer cells of tumor-bearing nude mice. However, 5-Fu conventional dose chemotherapy decreased the VEGF level, while capecitabine conventional dose chemotherapy increased the levels of VEGF and PDGF.

\section{Discussion}

5-FU-based chemotherapy is a first-line treatment for a variety of malignant tumors including gastric cancer $(21,22)$. Capecitabine is a precursor of 5-Fu drugs that is converted by the TP enzyme in tumor cells and has antitumor effects. As an oral agent, 5-Fu is more suitable for metronomic chemotherapy with a frequent low-dose pattern that has obvious advantages over intravenous drugs (23). Metronomic chemotherapy has low toxicity, good compliance, and better efficacy and represents a new trend in tumor chemotherapy $(24,25)$. In the present study, 5-Fu and capecitabine were administered 

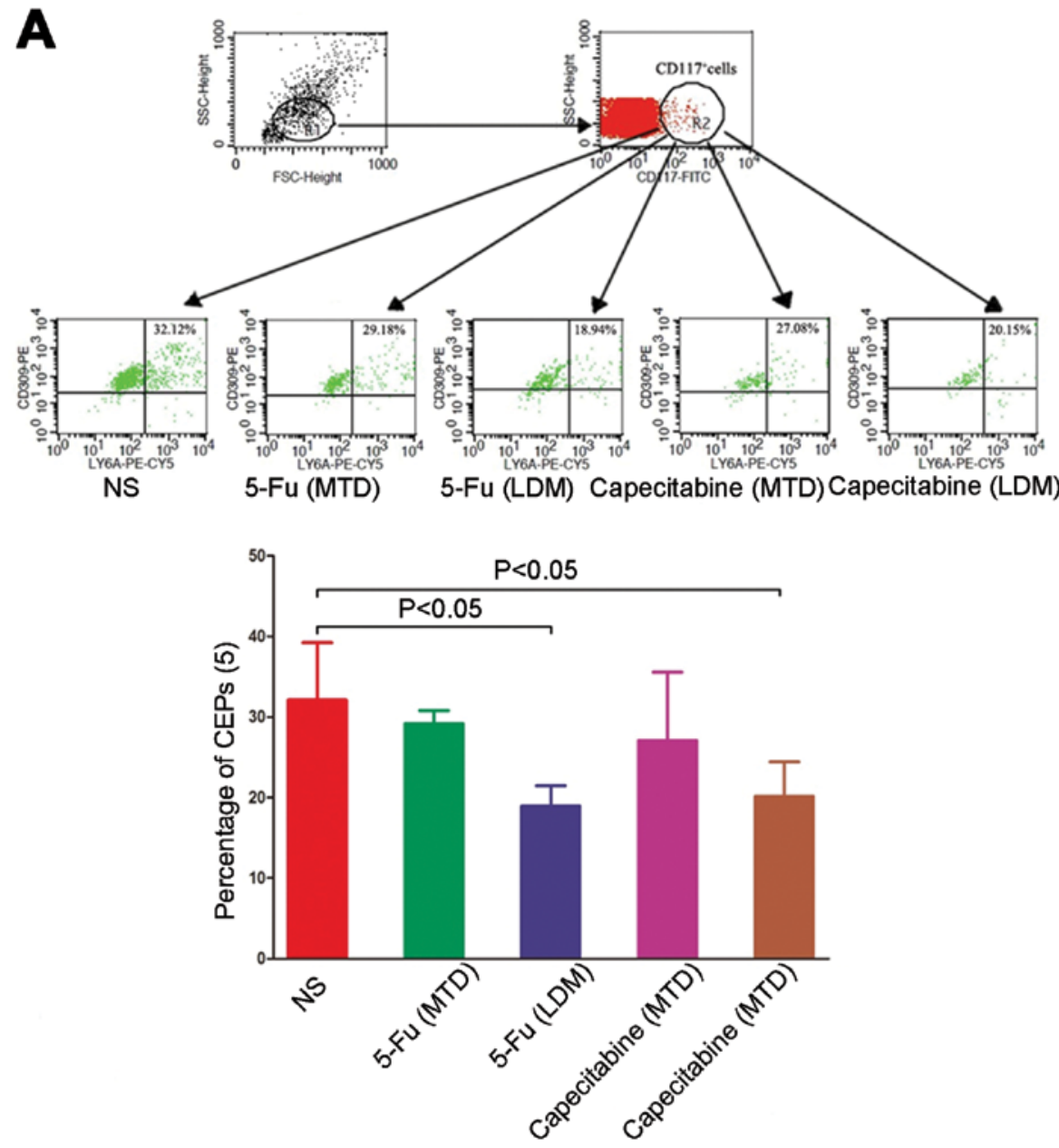

B

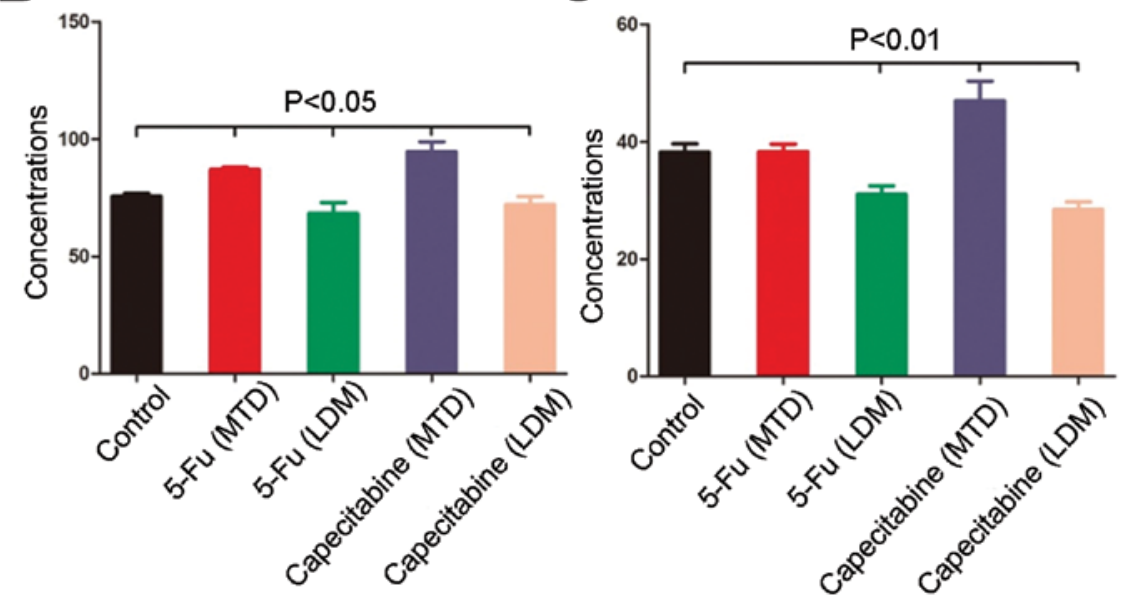

Figure 4. (A) CEPs in the peripheral blood of SGC-7901 gastric cancer-bearing nude mice. Angiogenesis-related factors in the peripheral blood of gastric cancer-bearing nude mice (B: VEGF; C: PDGF). CEPs, circulating endothelial progenitors.

in vitro, and the antitumor effects of 5-FU-based metronomic chemotherapy were observed and compared with conventional dose chemotherapy to verify the antitumor efficacy of metronomic chemotherapy.

The present study showed that metronomic 5-FU-based chemotherapy significantly inhibited the proliferation of gastric cancer cells in vitro and in vivo. The inhibitory effect was not weaker than that of conventional-dose chemotherapy.
The present results show that metronomic 5-FU-based chemotherapy inhibited the expression of secretory angiogenesis-related factors and vascular endothelial cell small tube formation, rather than having a cell toxicity effect that directly induced apoptosis and cell death, as compared with conventional dose chemotherapy. These results were similar to our preliminary study on colon cancer (9) with metronomic chemotherapy in other cancer types. Hackl et al (26) 
confirmed that irinotecan metronomic chemotherapy suspended transplanted colorectal cancer tumor growth and extended the survival of tumor-bearing nude mice. In animal experiments, Mainetti et al (27) found that CTX and adriamycin metronomic applications inhibited transplanted breast cancer growth and significantly reduced the VEGF level in a transplant tumor model. $\mathrm{Ng}$ et al (28) found that paclitaxel metronomic applications inhibited transplanted breast cancer growth by reducing the peripheral blood CEPs in tumor-bearing mice.

By inhibiting the proliferation and growth of tumors, we found that the body weight for the metronomic chemotherapy group was significantly higher than that of the standard chemotherapy group, indirectly indicating that metronomic chemotherapy had advantages regarding attenuating toxicity. For the first-line treatment of patients with gastric cancer, the PFS was 4-6 months. Existing drugs had limited efficacy, although the toxicities of combined chemotherapy made it less tolerable (29). Along with the emergence of new drugs such as taxanes and trastuzumab, first-line gastric cancer treatment prolonged the PFS to some extent, although toxicity remained the limiting factor $(30,31)$. Therefore, the use of metronomic chemotherapy for maintenance treatment has also been considered for gastric cancer, because of its low toxicity. Wu et al (32) reported that capecitabine metronomic chemotherapy suppressed the tumor growth of gastric cancer and had low toxicity. Fedele et al (33) treated metastatic breast cancer patients with oral Xeloda, showing a clinical benefit rate of $62 \%$, and grade 3-4 chemotherapy side effects were uncommon. In addition to cytotoxic effects, the inhibitory mechanism was also investigated. Instead of inhibiting tumor growth through cytotoxicity, metronomic chemotherapy attenuated the toxicity of conventional chemotherapy through the targeted inhibition of tumor angiogenesis.

Moreover, the level of the angiogenesis-related factor VEGF was increased in the conventional chemotherapy group to a certain extent, indicating that high-dose interval treatment with conventional chemotherapy may lead to the intermittent proliferation of remnant tumor cells. Bertolini et al (3) found that the CEP number and VEGF level were decreased with conventional chemotherapy but rebounded between chemotherapy cycles, suggesting that such high-dose intermittent treatment may promote tumor angiogenesis. Although the present study did not observe that the MVD was more intense with conventional chemotherapy than that of the control and metronomic chemotherapy groups, elevated VEGF levels in the peripheral blood and serum were demonstrated with no significant inhibition of peripheral blood CEPs. A 6-week drug treatment may not necessarily reflect an obvious difference in MVD, but significant differences in VEGF and PDGF provided clues for further investigation.

A variety of anti-VEGF drugs such as bevacizumab and aflibercept have demonstrated clear clinical efficacy $(34,35)$. However, bevacizumab did not demonstrate efficacy in the treatment of gastric cancer (10). Ramucizumab demonstrated positive results in second-line clinical investigations (11). These seem to contradict the clinical study results on at least two points: differences in treatment lines and mechanisms. The blockade of VEGFR or VEGF may lead to different treatment efficacies. The present study demonstrates the effect of metronomic chemotherapy on gastric cancer and focused not on tumor shrinkage, but on low-dose high-frequency tumor treatment. Metronomic chemotherapy played roles if the tumor load was relatively small (e.g., maintenance treatment after first-line PR treatment). However, it was not suitable for relatively large tumor loads. Second, the inhibition of tumor cell VEGF secretion demonstrated early molecular events by blocking the upstream tumor angiogenic effect, thus avoiding a standard-dose chemotherapy-induced rebound increase in VEGF secretion. This finding was similar to that of sorafenib treatment for the liver, as reported by Zhang et al (36). Those authors found that standard-dose chemotherapy and sorafenib treatment may lead to increased VEGF secretion from liver cancer cells (36); from the point of tumor angiogenesis, the addition of conventional dose chemotherapy induced a drugresistant mechanism.

As for drug economics, as compared with conventional dose chemotherapy, metronomic chemotherapy has decreased indirect costs and advantages given its high-potency ratio, including its low dose, high frequency and low toxicity and cumulative dose.

The current OS of patients with advanced gastric cancer was lower than that of breast and colorectal cancer. However, for the treatment of advanced gastric cancer, the status and value of metronomic chemotherapy has yet to be verified. As for advanced gastric cancer treatment, issues that remain to be addressed include, when to use metronomic chemotherapy, specifically, maintenance therapy; which patients; whether serum and tissue markers should be explored; the main roles of capecitabine (i.e., 5-Fu precursor plays a role in metronomic chemotherapy) in gastric cancer; and whether other oral medications (e.g., S-1) have similar effects.

\section{Acknowledgements}

The present study was supported by the National Science Foundation of China (81372645), the Shanghai Natural Science Foundation from the Municipal Government (13ZR1425900), the Shanghai Jiaotong University School of Medicine Science and Technology Foundation (13XJ10035), the Fong Shu Fook Tong Foundation and the National Key Clinical Discipline (Oncology).

\section{References}

1. Ferlay J, Shin HR, Bray F, Forman D, Mathers C and Parkin DM: Estimates of worldwide burden of cancer in 2008: GLOBOCAN 2008. Int J Cancer 127: 2893-2917, 2010.

2. Shah MA and Kelsen DP: Gastric cancer: a primer on the epidemiology and biology of the disease and an overview of the medical management of advanced disease. J Natl Compr Canc Netw 8: 437-447, 2010.

3. Bertolini F, Paul S, Mancuso P, et al: Maximum tolerable dose and low-dose metronomic chemotherapy have opposite effects on the mobilization and viability of circulating endothelial progenitor cells. Cancer Res 63: 4342-4346, 2003.

4. Hanahan D, Bergers G and Bergsland E: Less is more, regularly: metronomic dosing of cytotoxic drugs can target tumor angiogenesis in mice. J Clin Invest 105: 1045-1047, 2000.

5. Kamat AA, Kim TJ, Landen CN Jr, et al: Metronomic chemotherapy enhances the efficacy of antivascular therapy in ovarian cancer. Cancer Res 67: 281-288, 2007.

6. Man S, Bocci G, Francia G, et al: Antitumor effects in mice of low-dose (metronomic) cyclophosphamide administered continuously through the drinking water. Cancer Res 62: 2731-2735, 2002 . 
7. Orlando L, Cardillo A, Ghisini R, et al: Trastuzumab in combination with metronomic cyclophosphamide and methotrexate in patients with HER-2 positive metastatic breast cancer. BMC Cancer 6: 225, 2006.

8. Fakhrejahani E and Toi M: Antiangiogenesis therapy for breast cancer: an update and perspectives from clinical trials. Jpn J Clin Oncol 44: 197-207, 2014.

9. Shi H, Jiang J, Ji J, et al: Anti-angiogenesis participates in antitumor effects of metronomic capecitabine on colon cancer. Cancer Lett 349: 128-135, 2014.

10. Fuchs CS, Tomasek J, Yong CJ, et al: Ramucirumab monotherapy for previously treated advanced gastric or gastro-oesophageal junction adenocarcinoma (REGARD): an international, randomised, multicentre, placebo-controlled, phase 3 trial. Lancet 383: 31-39, 2014.

11. Ohtsu A, Shah MA, Van Cutsem E, et al: Bevacizumab in combination with chemotherapy as first-line therapy in advanced gastric cancer: a randomized, double-blind, placebo-controlled phase III study. J Clin Oncol 29: 3968-3976, 2011.

12. Kang BW, Kim JG, Kwon OK, Chung HY and Yu W: Non-platinum-based chemotherapy for treatment of advanced gastric cancer: 5-fluorouracil, taxanes, and irinotecan. World J Gastroenterol 20: 5396-5402, 2014.

13. Cunningham D, Starling N, Rao S, et al: Capecitabine and oxaliplatin for advanced esophagogastric cancer. N Engl J Med 358. 36-46, 2008.

14. Ryu MH and Kang YK: ML17032 trial: capecitabine/cisplatin versus 5-fluorouracil/cisplatin as first-line therapy in advanced gastric cancer. Expert Rev Anticancer Ther 9: 1745-1751, 2009.

15. Bang YJ, Kim YW, Yang HK, et al: Adjuvant capecitabine and oxaliplatin for gastric cancer after D2 gastrectomy (CLASSIC): a phase 3 open-label, randomised controlled trial. Lancet 379: 315-321, 2012.

16. Lee J, Lim do H, Kim S, et al: Phase III trial comparing capecitabine plus cisplatin versus capecitabine plus cisplatin with concurrent capecitabine radiotherapy in completely resected gastric cancer with D2 lymph node dissection: the ARTIST trial. J Clin Oncol 30: 268-273, 2012.

17. Shi M,Lou B, Ji J, et al: Synergistic antitumor effects of dasatinib and oxaliplatin in gastric cancer cells. Cancer Chemother Pharmacol 72: 35-44, 2013.

18. Kang WK, Park EK, Lee HS, et al: A biologically active angiogenesis inhibitor, human serum albumin-TIMP-2 fusion protein, secreted from Saccharomyces cerevisiae. Protein Expr Purif 53: 331-338, 2007

19. Zhang Q, Kang X, Yang B, Wang J and Yang F: Antiangiogenic effect of capecitabine combined with ginsenoside Rg3 on breast cancer in mice. Cancer Biother Radiopharm 23: 647-653, 2008.

20. Emoto M, Tachibana K, Iwasaki $\mathrm{H}$ and Kawarabayashi T: Antitumor effect of TNP-470, an angiogenesis inhibitor, combined with ultrasound irradiation for human uterine sarcoma xenografts evaluated using contrast color Doppler ultrasound. Cancer Sci 98: 929-935, 2007.

21. Li DH, Pan ZK, Ye F, An HX and Wu JX: S-1-based versus 5-FU-based chemotherapy as first-line treatment in advanced gastric cancer: a meta-analysis of randomized controlled trials. Tumour Biol 35: 8201-8208, 2014.
22. Sastre J, Garcia-Saenz JA and Diaz-Rubio E: Chemotherapy for gastric cancer. World J Gastroenterol 12: 204-213, 2006.

23. Bang YJ: Capecitabine in gastric cancer. Expert Rev Anticancer Ther 11: 1791-1806, 2011.

24. Gasparini G: Metronomic scheduling: the future of chemotherapy? Lancet Oncol 2: 733-740, 2001.

25. Loven D, Hasnis E, Bertolini F and Shaked Y: Low-dose metronomic chemotherapy: from past experience to new paradigms in the treatment of cancer. Drug Discov Today 18: 193-201, 2013.

26. Hackl C, Man S, Francia G, Milsom C, Xu P and Kerbel RS: Metronomic oral topotecan prolongs survival and reduces liver metastasis in improved preclinical orthotopic and adjuvant therapy colon cancer models. Gut 62: 259-271, 2013.

27. Mainetti LE, Rico MJ, Fernandez-Zenobi MV, et al: Therapeutic efficacy of metronomic chemotherapy with cyclophosphamide and doxorubicin on murine mammary adenocarcinomas. Ann Oncol 24: 2310-2316, 2013.

28. Ng SS, Sparreboom A, Shaked Y, et al: Influence of formulation vehicle on metronomic taxane chemotherapy: albumin-bound versus cremophor EL-based paclitaxel. Clin Cancer Res 12: 4331-4338, 2006.

29. Bilici A: Treatment options in patients with metastatic gastric cancer: current status and future perspectives. World J Gastroenterol 20: 3905-3915, 2014.

30. Gravalos C, Gomez-Martin C, Rivera F, et al: Phase II study of trastuzumab and cisplatin as first-line therapy in patients with HER2-positive advanced gastric or gastroesophageal junction cancer. Clin Transl Oncol 13: 179-184, 2011.

31. Guo Z, Wang X, Lin R, et al: Paclitaxel-based regimens as first-line treatment in advanced gastric cancer. J Chemother: Feb 18, 2014 (Epub ahead of print) doi: 1973947814Y0000000169.

32. Wu H, Xin Y, Xu C and Xiao Y: Capecitabine combined with (-)-epigallocatechin-3-gallate inhibits angiogenesis and tumor growth in nude mice with gastric cancer xenografts. Exp Ther Med 3: 650-654, 2012.

33. Fedele P, Marino A, Orlando L, et al: Efficacy and safety of low-dose metronomic chemotherapy with capecitabine in heavily pretreated patients with metastatic breast cancer. Eur J Cancer 48: 24-29, 2012.

34. Hurwitz H, Fehrenbacher L, Novotny W, et al: Bevacizumab plus irinotecan, fluorouracil, and leucovorin for metastatic colorectal cancer. N Engl J Med 350: 2335-2342, 2004.

35. Van Cutsem E, Tabernero J, Lakomy R, et al: Addition of aflibercept to fluorouracil, leucovorin, and irinotecan improves survival in a phase III randomized trial in patients with metastatic colorectal cancer previously treated with an oxaliplatin-based regimen. J Clin Oncol 30: 3499-3506, 2012.

36. Zhang W, Zhu XD, Sun HC, et al: Depletion of tumor-associated macrophages enhances the effect of sorafenib in metastatic liver cancer models by antimetastatic and antiangiogenic effects. Clin Cancer Res 16: 3420-3430, 2010. 